\title{
Condensable models of set theory
}

\author{
Ali Enayat ${ }^{1}(\mathbb{0}$ \\ Received: 6 November 2019 / Accepted: 30 June 2021 / Published online: 6 August 2021 \\ (c) The Author(s) 2021
}

\section{Abstract}

A model $\mathcal{M}$ of $\mathrm{ZF}$ is said to be condensable if $\mathcal{M} \cong \mathcal{M}(\alpha) \prec \mathbb{L}_{\mathcal{M}} \mathcal{M}$ for some "ordinal" $\alpha \in \operatorname{Ord}^{\mathcal{M}}$, where $\mathcal{M}(\alpha):=(\mathrm{V}(\alpha), \in)^{\mathcal{M}}$ and $\mathbb{L}_{\mathcal{M}}$ is the set of formulae of the infinitary logic $\mathbb{L}_{\infty, \omega}$ that appear in the well-founded part of $\mathcal{M}$. The work of Barwise and Schlipf in the 1970s revealed the fact that every countable recursively saturated model of ZF is cofinally condensable (i.e., $\mathcal{M} \cong \mathcal{M}(\alpha) \prec_{\mathbb{L}_{\mathcal{M}}} \mathcal{M}$ for an unbounded collection of $\alpha \in \operatorname{Ord}^{\mathcal{M}}$ ). Moreover, it can be readily shown that any $\omega$ nonstandard condensable model of ZF is recursively saturated. These considerations provide the context for the following result that answers a question posed to the author by Paul Kindvall Gorbow.

Theorem A. Assuming a modest set-theoretic hypothesis, there is a countable model $\mathcal{M}$ of ZFC that is both definably well-founded (i.e., every first order definable element of $\mathcal{M}$ is in the well-founded part of $\mathcal{M}$ ) and cofinally condensable. We also provide various equivalents of the notion of condensability, including the result below.

Theorem B. The following are equivalent for a countable model $\mathcal{M}$ of $\mathrm{ZF}$ :

(a) $\mathcal{M}$ is condensable.

(b) $\mathcal{M}$ is cofinally condensable.

(c) $\mathcal{M}$ is nonstandard and $\mathcal{M}(\alpha) \prec \mathbb{L}_{\mathcal{M}} \mathcal{M}$ for an unbounded collection of $\alpha \in \operatorname{Ord}^{\mathcal{M}}$.

Keywords Self-embedding $\cdot$ Recursive saturation · Nonstandard model of set theory $\cdot$ Infinitary languages

Mathematics Subject Classification Primary 03C62 · 03E30; Secondary 03H9

\section{Introduction}

By a classical theorem of Harvey Friedman [8], every countable nonstandard model $\mathcal{M}$ of ZF can be "shrunk" in the sense that $\mathcal{M}$ is isomorphic to a proper rank-initial segment of itself. Friedman's theorem was refined by Jean-Pierre Ressayre [15], who

$凶$ Ali Enayat

ali.enayat@gu.se

1 Department of Philosophy, Linguistics, and the Theory of Science, University of Gothenburg,

Gothenburg, Sweden 
constructed proper rank-initial self-embeddings of models of set theory that pointwise fix any prescribed rank-initial segment $\mathcal{M}(\alpha):=(\mathrm{V}(\alpha), \in)^{\mathcal{M}}$ of a model $\mathcal{M}$ of set theory determined by an "ordinal" $\alpha \in \operatorname{Ord}^{\mathcal{M}}$. More recently, Paul Kindvall Gorbow [9] extended Ressayre's work by carrying out a systematic study of the structure of fixed point sets of rank initial self-embeddings of models of set theory; and Zachiri McKenzie and the author [7], studied self-embeddings whose images are only required to be $\in$-initial segments of the ambient models.

By a general result of Gorbow [10, Theorem 7.2] if $\mathcal{M}$ is a countable nonstandard model of the fragment $\mathrm{KP}^{\mathcal{P}}+\Sigma_{1}^{\mathcal{P}}$-Separation of $\mathrm{ZF}$, then there are continuum-many proper rank-initial segments $\mathcal{N}$ of $\mathcal{M}$ that are isomorphic to $\mathcal{M}$, which makes it clear that there are continuum many such rank-initial segments $\mathcal{N}$ that are not of the form $\mathcal{M}(\alpha)$ for any "ordinal" $\alpha \in \operatorname{Ord}^{\mathcal{M}}$ (equivalently: $\operatorname{Ord}^{\mathcal{M}} \backslash \operatorname{Ord}^{\mathcal{N}}$ has no least element). It is also known that every consistent extension of ZF has a countable model $\mathcal{M}$ that is not isomorphic to any initial segment of itself that is of the form $\mathcal{M}(\alpha)$ for any $\alpha \in \operatorname{Ord}^{\mathcal{M}}{ }^{1}$ On the other hand, the pioneering work of Barwise and Schlipf on recursively saturated models in the 1970 s revealed a wealth of countable models $\mathcal{M}$ of set theory such that $\mathcal{M} \cong M(\alpha) \prec \mathcal{M}$ for many $\alpha \in \operatorname{Ord}^{\mathcal{M}}$, as indicated by the following theorem.

Theorem 1.1 (Barwise and Schlipf) Let Mbe a countable recursively saturated model of $\mathrm{ZF}$. Then there is some $\alpha \in \operatorname{Ord}^{\mathcal{M}}$ such that $\mathcal{M} \cong \mathcal{M}(\alpha) \prec \mathcal{M}$. Moreover, the collection of such $\alpha$ s is unbounded in $\operatorname{Ord}^{\mathcal{M}}$. $^{2}$

Motivated by the above theorem, we say that a model $\mathcal{M}$ of $\mathrm{ZF}$ is condensable if there is some $\alpha \in \operatorname{Ord}^{\mathcal{M}}$ such that $\mathcal{M} \cong \mathcal{M}(\alpha) \prec \mathbb{L}_{\mathcal{M}} \mathcal{M}$, where $\mathcal{M}(\alpha):=(\mathrm{V}(\alpha), \in)^{\mathcal{M}}$ and $\mathbb{L}_{\mathcal{M}}$ consists of formulae of the infinitary logic $\mathbb{L}_{\infty, \omega}$ that appear in the well-founded part of $\mathcal{M}$. Note that for an $\omega$-nonstandard model $\mathcal{M}$ of $\mathrm{ZF}, \mathbb{L}_{\mathcal{M}}$ is just the collection of (finitary) first order formulae, so the condition $\mathcal{M}(\alpha) \prec_{\mathbb{L}_{\mathcal{M}}} \mathcal{M}$ is equivalent to $\mathcal{M}(\alpha) \prec \mathcal{M}$ for $\omega$-nonstandard models $\mathcal{M}$ (and in particular for recursively saturated models $\mathcal{M}$ ). Theorem 1.1 provides the background for a question posed in an e-mail to the author (June 14, 2019).

Question 1.2 (Gorbow) Is there an $\omega$-standard model $\mathcal{M}$ of ZF such that the collection of $\alpha \in \operatorname{Ord}^{\mathcal{M}}$ satisfying $\mathcal{M} \cong \mathcal{M}(\alpha) \prec \mathcal{M}$ is unbounded in $\operatorname{Ord}^{\mathcal{M}}$ ?

In this article we establish Theorem A of the abstract (in Sect. 2) and a strengthening of Theorem B of the abstract (in Sect. 3). Theorem A yields a (strong) positive answer to Gorbow's question. Theorem B, on the other hand, provides various characterizations of condensability over the family of countable models of ZF.

\footnotetext{
1 This follows from the following facts: (1) Every countable extension of ZF has a Paris model, i.e., a model every ordinal of which is pointwise definable; (2) No Paris model of ZF can be rank extended to a model of Kripke-Platek set theory that has a first new ordinal; see Theorems 1.2 and 3.11 of [6].

2 Theorem 1.1 can be readily derived from the following two key results of Barwise and Schlipf: (a) Every resplendent model of ZF is cofinally condensable [17, Corollary 3.3]. (b) Every countable recursively saturated model is resplendent [2]. It is worth pointing out that the assumption of countability in Theorem 1.1 cannot be dropped since it is well-known that every consistent extension of ZF has an $\aleph_{1}$-like recursively saturated model (as elaborated in Remark 4.7). On the other hand, in light of the resplendence property of special models (attributed to Chang and Moschovakis in [2][Example 2.3]), every saturated model of ZF is cofinally condensable (it is well-known that ZFC proves that a saturated model of ZF of cardinality $\kappa$ exists iff $\mathrm{ZF}$ is consistent and $\kappa$ is an uncountable cardinal such that $\kappa^{<\kappa}=\kappa$ ).
} 


\section{Preliminaries}

In this section we collect the basic definitions, notations, conventions, and results that will be used in the statements and proofs of our main results in Sects. 2 and 3.

Definition 2.1 (Models, languages, and theories) Models will be represented by calligraphic fonts $(\mathcal{M}, \mathcal{N}$, etc. $)$ and their universes will be represented by the corresponding roman fonts $\left(M, N\right.$, etc.). In the definitions below, $\mathcal{M}$ is a model of $\mathrm{ZF}$ and $\in^{\mathcal{M}}$ is the membership relation of $\mathcal{M}$.

(a) $\operatorname{Ord}^{\mathcal{M}}$ is the class of "ordinals" of $\mathcal{M}$, i.e., $\operatorname{Ord}^{\mathcal{M}}:=\{m \in M: \mathcal{M} \models \operatorname{Ord}(m)\}$, where $\operatorname{Ord}(x)$ expresses " $x$ is transitive and is well-ordered by $\in$ ". More generally, given a class $\mathrm{D}$ whose defining formula is $\delta(x), \mathrm{D}^{\mathcal{M}}:=\{m \in M: \mathcal{M} \models \delta(m)\}$.

(b) $\mathcal{M}$ is nonstandard if $\epsilon^{\mathcal{M}}$ is ill-founded (equivalently: if (Ord, $\left.\epsilon\right)^{\mathcal{M}}$ is ill-founded). $\mathcal{M}$ is $\omega$-nonstandard if $(\omega, \in)^{\mathcal{M}}$ is ill-founded.

(c) For $c \in M, \operatorname{Ext}_{\mathcal{M}}(c)$ is the $\mathcal{M}$-extension of $c$, i.e., $\operatorname{Ext}_{\mathcal{M}}(c):=\{m \in M: m \in \mathcal{M}$ $c$. We say that a subset $X$ of $M$ is coded in $\mathcal{M}$ if there is some $c \in M$ such that $\operatorname{Ext}_{\mathcal{M}}(c)=X$. For $A \subseteq M, \operatorname{Cod}_{A}(\mathcal{M})$ will denote the collection of sets of the form $A \cap \operatorname{Ext}_{\mathcal{M}}(c)$, where $c \in M$.

(d) Given a model $\mathcal{N}$ of $\mathrm{ZF}$, we write $\mathcal{M} \subseteq$ rank $\mathcal{N}$ (read as: $\mathcal{M}$ is rank-extended by $\mathcal{M}$, or $\mathcal{M}$ is a rank-initial segment of $\mathcal{N}$ ), to indicate that $\mathcal{M}$ is a submodel of $\mathcal{N}$ such that $\rho^{\mathcal{M}}(x)>\rho^{\mathcal{M}}(m)$ whenever $x \in N \backslash M$ and $m \in M$, where $\rho(x)$ is the usual ordinal-valued rank function of set theory.

(e) The well-founded part of $\mathcal{M}$, denoted $\operatorname{WF}(\mathcal{M})$, consists of all elements $m$ of $\mathcal{M}$ such that there is no infinite sequence $\left\langle a_{n}: n<\omega\right\rangle$ with $m=a_{0}$ and $a_{n+1} \in^{\mathcal{M}} a_{n}$ for all $n \in \omega$. Given $m \in M$, we say that $m$ is a nonstandard element of $\mathcal{M}$ if $m \notin \mathrm{WF}(\mathrm{M})$. We denote the submodel of $\mathcal{M}$ whose universe is $\operatorname{WF}(\mathcal{M})$ by $\mathcal{W} \mathcal{F}(\mathcal{M})$. It is wellknown that if $\mathcal{M}$ is a model of $\mathrm{ZF}$, then $\mathcal{W} \mathcal{F}(\mathcal{M}) \subseteq$ rank $\mathcal{M}$, and $\mathcal{W} \mathcal{F}(\mathcal{M})$ satisfies KP (Kripke-Platek set theory) [1][Chapter II, Theorem 8.4]. Also note that if $\mathcal{M} \subseteq$ rank $\mathcal{N}$, where $\mathcal{M}$ is nonstandard, then $\mathcal{M}$ and $\mathcal{N}$ share the same well-founded part.

- It is important to bear in mind that we will identify $\mathcal{W} \mathcal{F}(\mathcal{M})$ with its transitive collapse.

(f) $\mathrm{o}(\mathcal{M})(\mathrm{read}$ as: the ordinal of $\mathcal{M})$ is the supremum of all ordinals that appear in the well-founded part of $\mathcal{M}$.

(g) Let $\mathcal{L}_{\text {set }}$ be the usual vocabulary $\{=, \in\}$ of set theory. In this paper we use $\mathbb{L}_{\infty, \omega}$ to denote the language using the vocabulary $\mathcal{L}_{\text {set }}$ that allows conjunctions and disjunctions of sets (but not proper classes) of formulae, subject to the restriction that such infinitary formulae have at most finitely many free variables. Given a set $\Psi$ of formulae, we denote such conjunctions and disjunctions respectively as $\bigwedge \Psi$ and $\bigvee \Psi$.

- In the interest of efficiency, we will treat disjunctions and universal quantification as defined notions.

(h) $\mathbb{L}_{\delta, \omega}$ is the sublanguage of $\mathbb{L}_{\infty, \omega}$ that allows conjunctions and disjunctions of sets of formulae of cardinality less than $\delta$. Note that $\mathbb{L}_{\omega, \omega}$ is none other than the usual first 
order language of set theory, and that in general the language $\mathbb{L}_{\delta, \omega}$ only uses finite strings of quantifiers (as indicated by the $\omega$ in the subscript). A full treatment can be found in [1, Chapter III].

(i) Given $\mathbb{L} \subseteq \mathbb{L}_{\infty, \omega}$, and $\mathcal{L}_{\text {set-structures }} \mathcal{N}_{1}$ and $\mathcal{N}_{2}$, we write $\mathcal{N}_{1} \prec \mathbb{L} \mathcal{N}_{2}$ to indicate that $\mathcal{N}_{1}$ is a submodel of $\mathcal{N}_{2}$ and for every $\varphi\left(x_{1}, \ldots, x_{n}\right) \in \mathbb{L}$, and any $n$-tuple $\left(a_{1}, \ldots, a_{n}\right)$ from $N_{1}$, we have:

$$
\mathcal{N}_{1} \models \varphi\left(a_{1}, \ldots, a_{n}\right) \text { iff } \mathcal{N}_{2} \models \varphi\left(a_{1}, \ldots, a_{n}\right) .
$$

(j) $\mathbb{L}_{\mathcal{M}}=\mathbb{L}_{\infty, \omega} \cap \mathrm{WF}(\mathcal{M})$. Note that if $M$ is countable, $\mathbb{L}_{\mathcal{M}}=\mathbb{L}_{\omega_{1}, \omega} \cap \operatorname{WF}(\mathcal{M})$.

(k) Given $\mathbb{L} \subseteq \mathbb{L}_{\infty, \omega}, \mathrm{Th}_{\mathbb{L}}(\mathcal{M})$ is the set of sentences (closed formulae) of $\mathbb{L}$ that hold in $\mathcal{M}$, and $\mathrm{ZF}(\mathbb{L})$ is the natural extension of $\mathrm{ZF}$ in which the scheme Sep of separation and Coll of collection are extended to the schemes $\operatorname{Sep}(\mathbb{L})$ and $\operatorname{Coll}(\mathbb{L})$ to allow formulae in $\mathbb{L}$ to be used for "separating" and " collecting" (respectively).

(l) For $\varphi \in \mathbb{L}_{\infty, \omega}$, the depth of $\varphi$, denoted Depth $(\varphi)$, is the ordinal defined recursively by the following clauses:

(1) $\operatorname{Depth}(\varphi)=0$, if $\varphi$ is an atomic formula.

(2) $\operatorname{Depth}(\varphi)=\operatorname{Depth}(\psi)+1$, if $\varphi=\neg \psi$.

(3) $\operatorname{Depth}(\varphi)=\operatorname{Depth}(\psi)+1$, if $\varphi=\exists x \psi$.

(4) $\operatorname{Depth}(\varphi)=\sup \{\operatorname{Depth}(\psi)+1: \psi \in \Psi\}$, if $\varphi=\bigwedge \Psi$.

- Within KP, one can code each formula $\varphi \in \mathbb{L}_{\infty, \omega}$ with a set $\ulcorner\varphi\urcorner$ as in Chapter 3 of [1], but in the interest of better readability we will often identify a formula with its code. This coding allows us to construe statements such as $\varphi \in \mathbb{L}_{\infty, \omega}$ and $\operatorname{Depth}(\varphi)=\alpha$ as statements in the first order language of set theory. It is easy to see that the collection $\mathrm{D}(\alpha)$ of (codes of) $\mathbb{L}_{\infty, \omega}$-formulae whose depth is less than $\alpha$ forms a set in $\mathrm{ZF}$ for all ordinals $\alpha$ since a simple induction shows that for a sufficiently large $k \in \omega, \mathrm{D}(\alpha) \subseteq \mathrm{V}(\omega+k \alpha)$ for each ordinal $\alpha$. This makes it clear that $\mathbb{L}_{\mathcal{M}}=\bigcup_{\alpha \in \mathrm{o}(\mathcal{M})} \mathrm{D}^{\mathcal{M}}(\alpha)$.

Definition 2.2 Suppose $\mathcal{M}$ is a model of $\mathrm{ZF}$, and $S \subseteq M$.

(a) $S$ is separative (over $\mathcal{M}$ ) if $(\mathcal{M}, S)$ satisfies the separation scheme $\operatorname{Sep}(\mathrm{S})$ in the extended language that includes a fresh predicate $S$ (interpreted by $S$ ).

(b) $S$ is collective (over $\mathcal{M}$ ) if $(\mathcal{M}, S$ ) satisfies the collection scheme Coll(S) in the extended language that includes a fresh predicate $S$ (interpreted by $S$ ).

(c) $S$ is amenable (over $\mathcal{M}$ ) if $S$ is both separative and collective. In other words, $S$ is amenable if $(\mathcal{M}, S)$ satisfies the replacement scheme $\operatorname{Repl}(S)$ in the extended language that includes a fresh predicate $S$ (interpreted by $S$ ).

(d) For $\alpha \in \operatorname{Ord}^{\mathcal{M}}, S$ is an $\alpha$-satisfaction class (over $\mathcal{M}$ ) if $S$ correctly decides the truth of atomic sentences, and $S$ satisfies Tarski's compositional clauses of a truth predicate for $\mathrm{D}^{\mathcal{M}}(\alpha)$-sentences (see below for the precise definition). $S$ is an $\infty$-satisfaction class over $\mathcal{M}$, if $S$ is an $\alpha$-satisfaction class over $\mathcal{M}$ for every $\alpha \in \operatorname{Ord}^{\mathcal{M}}$. 
We elaborate the meaning of (d) above. Reasoning within KP , for each object $a$ in the universe of sets, let $c_{a}$ be a constant symbol denoting $a$ (where the map $a \mapsto c_{a}$ is $\left.\Delta_{1}\right)$, and let $\operatorname{Sent}^{+}(\alpha, x)$ be the set-theoretic formula (with an ordinal parameter $\alpha$ and the free variable $x$ ) that defines the proper class of sentences of the form $\varphi\left(c_{a_{1}}, \ldots, c_{a_{n}}\right)$, where $\varphi\left(x_{1}, \ldots, x_{n}\right) \in \mathrm{D}(\alpha)$ (the superscript + on $\operatorname{Sent}^{+}(\alpha, x)$ indicates that $x$ is a sentence in the language augmented with the indicated proper class of constant symbols). Then $S$ is an $\alpha$-satisfaction class over $\mathcal{M}$ if $(\mathcal{M}, S) \models \operatorname{Sat}(\alpha, S)$, where $\operatorname{Sat}(\alpha, S)$ is the (universal generalization of) the conjunction of the axioms $(I)$ through $(I V)$ below.

(I) $\quad\left(\left(\mathrm{S}\left(\left\ulcorner c_{a}=c_{b}\right\urcorner\right) \leftrightarrow a=b\right) \wedge\left(\mathrm{S}\left(\left\ulcorner c_{a} \in c_{b}\right\urcorner\right) \leftrightarrow a \in b\right)\right)$.

(II ) $\left(\operatorname{Sent}^{+}(\alpha, \varphi) \wedge(\varphi=\neg \psi)\right) \rightarrow(\mathrm{S}(\varphi) \leftrightarrow \neg \mathrm{S}(\psi))$.

(III $)\left(\operatorname{Sent}^{+}(\alpha, \varphi) \wedge(\varphi=\bigwedge \Psi)\right) \rightarrow(\mathrm{S}(\varphi) \leftrightarrow \forall \psi \in \Psi \mathrm{S}(\psi))$.

$(I V)\left(\operatorname{Sent}^{+}(\alpha, \varphi) \wedge(\varphi=\exists x \psi(x))\right) \rightarrow\left(\mathrm{S}(\varphi) \leftrightarrow \exists x \mathrm{~S}\left(\psi\left(c_{x}\right)\right)\right)$.

(e) For $\alpha<\mathrm{o}(\mathcal{M}), S$ is the $\alpha$-satisfaction class over $\mathcal{M}$, if $S$ is the usual Tarskian satisfaction class for formulae in $\mathbb{L}_{\mathcal{M}}$ of depth less than $\alpha$, i.e., the unique $\alpha$-satisfaction class $S$ over $\mathcal{M}$ such that $S$ satisfies:

(V) $\forall x\left(\mathrm{~S}(x) \rightarrow \operatorname{Sent}^{+}(\alpha, x)\right)$.

- In the interest of a lighter notation we will often confuse constant symbols in formulae with their denotations, e.g., we will write $\varphi(a)$ instead of $\varphi\left(c_{a}\right)$.

The following proposition is immediately derivable from the definitions involved.

Proposition 2.3 If $S$ is an $\alpha$-satisfaction class over $\mathcal{M} \models \mathrm{KP}$ for some nonstandard ordinal $\alpha$ of $\mathcal{M}$, then for all $n$-ary formula $\varphi\left(x_{1}, \ldots, x_{n}\right)$ of $\mathbb{L}_{\mathcal{M}}$ and all $n$-tuples $\left(a_{1}, \ldots, a_{n}\right)$ from $M$, we have:

$$
\mathcal{M} \models \varphi\left(a_{1}, \ldots, a_{n}\right) \text { iff } \varphi\left(a_{1}, \ldots, a_{n}\right) \in S \text {. }
$$

In particular, for all sentences $\varphi$ of $\mathbb{L}_{\mathcal{M}}, \varphi \in S$ iff $\varphi \in \operatorname{Th}_{\mathbb{L}_{\mathcal{M}}}(\mathcal{M})$.

Remark 2.4 Reasoning within ZF, given any limit ordinal $\gamma,(\mathrm{V}(\gamma), \in)$ carries a separative $\gamma$-satisfaction class $S$ since we can take $S$ to be the Tarskian satisfaction class on $(\mathrm{V}(\gamma), \in)$ for formulae of depth less than $\gamma$. More specifically, the Tarski recursive construction/definition of truth works equally well in this more general context of infinitary languages since $(\mathrm{V}(\gamma), \in)$ forms a set. Observe that $(\mathrm{V}(\gamma), \in, S) \models \operatorname{Sep}(\mathrm{S})$ comes "for free" since for any $X \subseteq \mathrm{V}(\gamma)$ the expansion $(\mathrm{V}(\gamma), \in, X)$ satisfies the scheme of separation in the extended language. However, $S$ need not be collective, for example if the collection of first order definable elements of $(\mathrm{V}(\gamma), \in)$ is cofinal in $\gamma$.

Proposition 2.5 If $\mathcal{M} \models \mathrm{KP}$, then for each $\alpha \in \mathrm{o}(\mathcal{M})$ there is a formula $\operatorname{Sat}_{\alpha}(x) \in$ $\mathbb{L}_{\mathcal{M}}$ such that $\operatorname{Sat}_{\alpha}^{\mathcal{M}}(x)$ is the $\alpha$-satisfaction class over $\mathcal{M}$.

Proof The desired formula $\operatorname{Sat}_{\alpha}(x)$ is defined by the following recursion. A routine induction on $\alpha$ shows that $\operatorname{Sat}_{\alpha}(x)$ has the desired properties. One can also verify that $\operatorname{Sat}_{\alpha}(x) \in \mathrm{WF}(\mathcal{M})$ for $\alpha \in \mathrm{o}(\mathcal{M})$ by observing that the recursion defining $\operatorname{Sat}_{\alpha}(x)$ is a so-called $\Sigma_{1}$-recursion (recall that $\mathcal{W F}(\mathcal{M})$ satisfies $\mathrm{KP}$, and KP can handle constructions by $\Sigma_{1}$-recursion). 
- $\operatorname{Sat}_{1}(x):=\exists y \exists z\left[\left(\left(x=\left\ulcorner c_{y}=c_{z}\right\urcorner\right) \wedge(y=z)\right) \vee\left(\left(x=\left\ulcorner c_{y} \in c_{z}\right\urcorner\right) \wedge(y \in z)\right)\right]$.

- For $\alpha>1, \operatorname{Sat}_{\alpha}(x):=\left[(\operatorname{Depth}(x)=0) \wedge \operatorname{Sat}_{1}(x)\right] \vee$

$$
\bigvee_{0<\beta<\alpha}\left(\operatorname{Depth}(x)=\beta \wedge\left[\operatorname{Neg}_{\beta}(x) \vee \operatorname{Exist}_{\beta}(x) \vee \operatorname{Conj}_{\beta}(x)\right]\right),
$$

where:

$$
\begin{aligned}
& \operatorname{Neg}_{\beta}(x):=\exists y\left[(x=\ulcorner\neg y\urcorner) \wedge \neg \operatorname{Sat}_{\beta}(y)\right], \\
& \operatorname{Exist}_{\beta}(x):=\exists y\left[\exists v(x=\ulcorner\exists v y(v)\urcorner) \wedge \exists v \operatorname{Sat}_{\beta}\left(y\left(c_{v}\right)\right],\right. \text { and } \\
& \left.\operatorname{Conj}_{\beta}(x):=\exists y[(x=\ulcorner\bigwedge y\urcorner)) \wedge\left(\forall z \in y \operatorname{Sat}_{\beta}(z)\right)\right] .
\end{aligned}
$$

The following proposition will be called upon in the proofs of Theorems A and B.

Proposition 2.6 (Overspill) Suppose $\mathcal{M}$ is a nonstandard model of $\mathrm{ZF}$, and $S \subseteq M$ such that $S$ is separative over $\mathcal{M}$. Assume furthermore that there is a first order formula $\theta(x, \bar{y})$ in the language $\{\in, \mathrm{S}\}$ and some sequence of parameters $\bar{m} \in M$ such that $(\mathcal{M}, S) \models \theta(\alpha, \bar{m})$ for every $\alpha \in \mathrm{o}(\mathcal{M})$. Then there is a nonstandard $\gamma \in \operatorname{Ord}^{\mathcal{M}}$ such that $(\mathcal{M}, S) \models \theta(\gamma, \bar{m})$.

Proof Suppose not, and let $A:=\mathrm{WF}(\mathcal{M}) \cap \operatorname{Ord}^{\mathcal{M}}$. Then $A=\{x \in M:(\mathcal{M}, S) \models \theta$ $(x, \bar{m}) \wedge \operatorname{Ord}(x)\}$. Since $A$ is a bounded subset of $\operatorname{Ord}^{\mathcal{M}}, A$ is coded in $\mathcal{M}$ by $\operatorname{Sep}(\mathrm{S})$, and therefore has a supremum $\beta$ in $\mathcal{M}$. This is a contradiction since $(\beta, \in)^{\mathcal{M}}$ is well-founded, and yet $\beta \notin A$ since $A$ has no last element.

The following general versions of the elementary chains theorem of model theory, and of the reflection theorem of set theory will be called upon in the proof of Theorem B. The proofs of Proposition 2.7 is obtained by a routine adaptations of the usual proofs of the $\mathbb{L}_{\omega, \omega}$-version of the elementary chain theorem (e.g., as in [3][Theorem 3.1.9]).

Proposition 2.7 (Elementary Chains) Suppose $\mathbb{L} \subseteq \mathbb{L}_{\infty, \omega}$ where $\mathbb{L}$ is closed under subformulae; $(I, \triangleleft)$ is a linear order; $\left\langle\mathcal{M}_{i}: i \in I\right\rangle$ is an $\mathbb{L}$-elementary chain of structures (i.e., $\mathcal{M}_{i} \prec \mathbb{L} \mathcal{M}_{j}$ whenever $i \triangleleft j$ ); and $\mathcal{M}=\bigcup_{i \in I} \mathcal{M}_{i}$. Then $\mathcal{M}_{i} \prec \mathbb{L} \mathcal{M}$ for each $i \in I$.

Proposition 2.8 (Reflection) Suppose $\mathcal{M} \models \mathrm{ZF}\left(\mathbb{L}_{\mathcal{M}}\right)$, and for each $\varphi \in \mathbb{L}_{\mathcal{M}}$ where $\varphi$ is $n$-ary, let $\operatorname{Ref}_{\varphi}(\gamma)$ be the $\mathbb{L}_{\mathcal{M}}$-formula:

$$
\forall x_{1} \in \mathrm{V}(\gamma) \ldots \forall x_{n} \in \mathrm{V}(\gamma)\left[\varphi\left(x_{1}, \ldots, x_{n}\right) \longleftrightarrow \varphi^{\mathrm{V}(\gamma)}\left(x_{1}, \cdots, x_{n}\right)\right]
$$

Then for any $\alpha \in \mathrm{o}(\mathcal{M})$ there are arbitrarily large $\gamma \in \operatorname{Ord}^{\mathcal{M}}$ such that $\mathcal{M}(\gamma)$ reflects all formulae in $D^{\mathcal{M}}(\alpha)$, i.e., $\mathcal{M} \models \operatorname{Ref}_{\varphi}(\gamma)$ for all $\mathbb{L}_{\mathcal{M}}$-formulae $\varphi$ of depth less than $\alpha$. 
Proof We will take advantage of Proposition 2.5 to derive Proposition 2.8 from the usual finitary formulation of Reflection Theorem ${ }^{3}$. Given $\alpha \in \mathrm{o}(\mathcal{M})$, by Proposition 2.5, there is a formula $\operatorname{Sat}_{\alpha}(x) \in \mathbb{L}_{\mathcal{M}}$ such that $\operatorname{Sat}_{\alpha}^{\mathcal{M}}(x)$ is an $\alpha$-satisfaction class over $\mathcal{M}$. Let $S:=\operatorname{Sat}_{\alpha}^{\mathcal{M}}(x)$; note that since $\mathcal{M}$ is assumed to satisfy $\mathrm{ZF}\left(\mathbb{L}_{\mathcal{M}}\right), S$ is amenable over $\mathcal{M}$. Let $\operatorname{Sat}(\alpha, \mathrm{S})$ be the finitary formula that express " $\mathrm{S}$ is an $\alpha$ satisfaction class" (as in part (d) of Definition 2.2). By the amenability of $S$ over $\mathcal{M}$, we may invoke the usual finitary Reflection Theorem to obtain arbitrarily large $\gamma \in \mathrm{Ord}^{M}$ such that $(\mathcal{M}(\gamma), S \cap M(\gamma))$ reflects the formula $\psi(x, \mathrm{~S}):=\operatorname{Sat}(\alpha, \mathrm{S}) \wedge \mathrm{S}(x)$, i.e.,

$$
(\mathcal{M}, S) \models \forall x \in \mathrm{V}(\gamma)\left[\psi(x, \mathrm{~S}) \longleftrightarrow \psi^{\mathrm{V}(\gamma)}(x, \mathrm{~S})\right] .
$$

It is now easy to verify, using Proposition 2.3, that this choice of $\gamma$ satisfies the conclusion of Proposition 2.8.

\section{Proof of Theorem A}

The proof of Theorem A is based on some preliminary results. The reader may wish to skip the proofs of these results in the first reading to be able to better see the overall structure of the proof of Theorem A.

Lemma 3.1 Suppose $\mathcal{M}$ and $\mathcal{N}$ are countable nonstandard models of ZF with the same well-founded part $W$, and let $\mathbb{L}:=\mathbb{L}_{\mathcal{M}}=\mathbb{L}_{\mathcal{N}}$. Then $\mathcal{M}$ and $\mathcal{N}$ are isomorphic if the following three conditions are satisfied:

(I) $\operatorname{Cod}_{W}(\mathcal{M})=\operatorname{Cod}_{W}(\mathcal{N})$.

$(I I) \mathrm{Th}_{\mathbb{L}}(\mathcal{M})=\mathrm{Th}_{\mathbb{L}}(\mathcal{N})$

(I I I) For some nonstandard ordinals $\tau_{M}$ of $\mathcal{M}$ and $\tau_{N}$ of $\mathcal{N}$ there are $S_{M} \subseteq M$ and $S_{N} \subseteq N$ such that $S_{M}$ is a separative $\tau_{M}$-satisfaction class over $\mathcal{M}$, and $S_{N}$ is a separative $\tau_{N}$-satisfaction class over $\mathcal{N}$.

Proof The isomorphism between $\mathcal{M}$ and $\mathcal{N}$ can be built by a routine back-and-forth construction once we establish Claim 3.1.1 below, for which we introduce the following convention:

- Given an $n$-tuple $\bar{a}=\left(a_{0}, \ldots, a_{n-1}\right)$ from $M$ (where $\left.n \in \omega\right)$, and an $n$-tuple $\bar{b}=\left(b_{0}, \ldots, b_{n-1}\right)$ from $N$, we write $\bar{a} \sim \bar{b}$ as a shorthand for the following statement, where $\bar{x}$ is an $n$-tuple:

for each $n$-ary formulae $\varphi(\bar{x})$ of $\mathbb{L}, \varphi(\bar{a}) \in S_{M}$ iff $\varphi(\bar{b}) \in S_{N}$.

Note that by Proposition 2.3, $\bar{a} \sim \bar{b}$ iff for all $n$-ary formulae $\varphi(\bar{x})$ of $\mathbb{L}, \mathcal{M} \models \varphi(\bar{a})$ iff $\mathcal{N}=\varphi(\bar{a})$. In particular, $\varnothing \sim \varnothing$, by condition $(I I)$ of the lemma, where $\varnothing$ is the "0-tuple", i.e., the empty sequence.

3 The Reflection Theorem is often formulated as a theorem scheme of ZF (e.g., as in [12]), but it is wellknown that the proof strategy of the Reflection Theorem applies equally well to the extension $\mathrm{ZF}(\mathcal{L})$ of $\mathrm{ZF}$ (where $\mathcal{L}$ extends the language of set theory) in which the schemes of separation and collection are extended to $\mathcal{L}$-formulae. 
3.1.1. Claim. Suppose $\bar{a} \sim \bar{b}$. Then:

(i) For every $a \in M$ there is some $b \in N$ such that $(\bar{a}, a) \sim(\bar{b}, b)$.

(ii) For every $b \in N$ there is some $a \in M$ such that $(\bar{a}, a) \sim(\bar{b}, b)$.

By symmetry it suffices to verify part $(i)$ of Claim 3.1.1. Observe that since $\mathcal{M}$ and $\mathcal{N}$ share the same well-founded part $W$, we can fix an ordinal $\eta$ such that $\eta=\mathrm{o}(\mathcal{M})=$ $\mathrm{o}(\mathcal{N})$, and $N(\alpha)=M(\alpha)$ for all $\alpha<\eta$.

Given $a \in M$, let

$X:=\left\{\varphi(\bar{v}, v): \varphi(\bar{v}, v)\right.$ is an $(n+1)$-ary formula of $\mathbb{L}$, and $\left.\varphi(\bar{a}, a) \in S_{M}\right\}$.

A routine argument shows that $X \in \operatorname{Cod}_{W}(\mathcal{M})$ (using the assumption that $S_{M}$ is a separative $\tau_{M}$-satisfaction class over $\mathcal{M}$ and $\tau_{M}$ is a nonstandard ordinal of $\mathcal{M}$ ). So by assumption $(I)$ of Lemma 3.1, $X \in \operatorname{Cod}_{W}(\mathcal{N})$. Hence there is some $c \in M$ such that $X=W \cap \operatorname{Ext}_{\mathcal{N}}(c)$. For any $\alpha \in \operatorname{Ord}^{\mathcal{M}}$, consider the elements $c_{\alpha}$ and $d_{\alpha}$ of $N$, such that the following holds in $\mathcal{N}$ :

$$
c_{\alpha}=\{x \in c: x \in \mathrm{V}(\alpha)\} \text { and } d_{\alpha}=\{x \in \mathrm{V}(\alpha): x \notin c\} .
$$

Then for each $\alpha<\eta$, both $c_{\alpha}$ and $d_{\alpha} \in W$. Also, in light of our convention of identifying $W$ with its transitive collapse, for each $w \in W$ we have:

$$
w=\operatorname{Ext}_{\mathcal{M}}(w)=\operatorname{Ext}_{\mathcal{N}}(w)
$$

The choice of $c_{\alpha}$ and $d_{\alpha}$ together with the compositional properties of $S_{M}$ allows us to conclude:

(1) For all $\alpha \in \eta \overbrace{\left(\exists x\left(\bigwedge_{\varphi(\bar{v}, v) \in c_{\alpha}} \varphi(\bar{a}, x)\right) \wedge\left(\bigwedge_{\varphi(\bar{v}, v) \in d_{\alpha}} \neg \varphi(\bar{a}, x)\right)\right)}^{\psi_{\alpha}(\bar{a})} \in S_{M}$.

Observe that $\psi_{\alpha}(\bar{x})$ is a formula of $\mathbb{L}$. Putting (1) together with the assumption $\bar{a} \sim \bar{b}$ yields $\psi_{\alpha}(\bar{b}) \in S_{N}$, i.e.,

(2) For all $\left.\alpha \in \eta\left(\exists x\left(\bigwedge_{\varphi(\bar{v}, v) \in c_{\alpha}} \varphi(\bar{b}, x)\right) \wedge\left(\bigwedge_{\varphi(\bar{v}, v) \in d_{\alpha}} \neg \varphi(\bar{b}, x)\right)\right)\right) \in S_{N}$.

The key observation at this point is that there is a first order formula $\theta(\mathrm{S}, x, y, \bar{z})$ in the language of set theory augmented with the predicate $S$ such that (2) can be re-expressed as:

(3) For all $\alpha \in \eta,\left(\mathcal{N}, S_{N}\right)=\theta(\mathrm{S}, \alpha, c, \bar{b})$.

By invoking Overspill (Proposition 2.6) in the expanded structure $\left(\mathcal{N}, S_{N}\right)$, there is some nonstandard ordinal $\gamma$ of $\mathcal{N}$ such $\left(\mathcal{N}, S_{N}\right) \models \theta(\mathrm{S}, \gamma, c, \bar{b})$, i.e.,

(4) $\left.\left(\mathcal{N}, S_{N}\right)=\mathrm{S}\left(\exists x\left(\bigwedge_{\varphi(\bar{v}, v) \in c_{\gamma}} \varphi(\bar{b}, x)\right) \wedge\left(\bigwedge_{\varphi(\bar{v}, v) \in d_{\gamma}} \neg \varphi(\bar{b}, x)\right)\right)\right)$.

By coupling (4) together with the assumption that $\left(\mathcal{N}, S_{N}\right)$ satisfies condition $(I V)$ of $\operatorname{Sat}\left(\tau_{N}, S\right.$ ) (as in Definition 2.2), the existential statement deemed true in (4) by the interpretation $S_{N}$ of $\mathrm{S}$ is witnessed by some $b \in N$. It should be clear that this is the desired element $b \in N$, i.e., $(\bar{a}, a) \sim(\bar{b}, b)$. This concludes the proof of Claim 3.1.1, and therefore of Lemma 3.1. 
We now present an easy lemma (Lemma 3.2), and an old theorem of Hutchinson (Theorem 3.3); they will allow us to arrange the hypotheses of Lemma 3.1 in the proof of Theorem A.

Lemma 3.2 (ZFC) Let $\lambda$ be a strongly inaccessible cardinal, $S \subseteq \mathrm{V}(\lambda)$, and let

$$
C:=\{\delta<\lambda:(\mathrm{V}(\delta), \in, S \cap \mathrm{V}(\delta)) \prec(\mathrm{V}(\lambda), \in, S)\}
$$

Then $C$ is closed and unbounded in $\lambda$.

Proof $C$ is clearly closed by the elementary chain theorem, so we will concentrate on demonstrating the unboundedness of $C$. Fix a well-ordering $\triangleleft$ of $\mathrm{V}(\lambda)$, and for any $A \subseteq \mathrm{V}(\lambda)$, let $\mathcal{H}(A)$ be the submodel of $(\mathrm{V}(\lambda), \in, S)$ whose universe $H(A)$ consists of the elements of $\mathrm{V}(\lambda)$ that are first order definable in the expanded structure $(\mathrm{V}(\lambda), \in, S, \triangleleft, a)_{a \in A}$. Clearly $|H(A)|=\min \left\{\aleph_{0},|A|\right\}$, and by Tarski's test $\mathcal{H}(A) \prec$ $(\mathrm{V}(\lambda), \in, S)$. Given an ordinal $\alpha<\lambda$, we will exhibit $\beta$ such that $\alpha \leq \beta \in C$. To this end, consider the sequence of models $\left\langle\mathcal{M}_{n}: n \in \omega\right\rangle$ and sequence of ordinals $\left\langle\alpha_{n}: n \in \omega\right\rangle$ defined by the following recursive clauses:

- $\alpha_{0}:=\alpha$, and $\mathcal{M}_{0}:=\mathcal{H}\left(\alpha_{0}\right)$.

- $\alpha_{n+1}:=\sup \left\{\beta<\kappa: \beta \in M_{n}\right\}$, and $\mathcal{M}_{n+1}:=\mathcal{H}\left(\mathrm{V}\left(\alpha_{n+1}\right)\right)$.

The strong inaccessibility of $\lambda$ guarantees that $\mathcal{M}_{n}$ and $\alpha_{n}$ are well-defined for each $n \in \omega$, and that $\left\{\alpha_{n}: n \in \omega\right\}$ is bounded in $\lambda$. Let $\beta:=\sup \left\{\alpha_{n}: n \in \omega\right\}$. It is routine to verify that $\alpha \leq \beta \in C$.

The following theorem was established by Hutchinson [11] using the omitting types theorem. As shown in [4, Theorem 2.12] this result can also be proved for models of ZFC using generic ultrapowers.

Theorem 3.3 (Hutchinson) Suppose $\lambda$ is a regular cardinal in a countable model $\mathcal{K}$ of ZF. Then there is an elementary extension $\mathcal{K}^{*}$ of $\mathcal{K}$ satisfying the following two properties:

(a) $\mathcal{K}^{*}$ does not "perturb" any ordinal of $\mathcal{K}$ that is below $\lambda$, i.e., if $\mathcal{K} \models \alpha \in \lambda$, then $\operatorname{Ext}_{\mathcal{K}}(\alpha)=\operatorname{Ext}_{\mathcal{K}^{*}}(\alpha)$

(b) $\operatorname{Ext}_{\mathcal{K}^{*}}(\lambda) \backslash \operatorname{Ext}_{\mathcal{K}}(\lambda)$, when ordered by $\in^{\mathcal{K}^{*}}$, has no first element (under the ordering $\left.\in^{\mathcal{K}^{*}}\right)$

Remark 3.4 Condition (a) of Theorem 3.3 ensures that if $k \in K$ and $\mathcal{K} \models|k|<\lambda$, then $\mathcal{K}$ does not perturb $k$. To see this, choose $f$ and $\alpha$ in $K$ such that:

$$
\mathcal{K}=" \alpha \in \lambda \text { and } f: \alpha \rightarrow k \text { and } f \text { is a bijection". }
$$

Then since $\mathcal{K} \prec \mathcal{K}^{*}, \operatorname{Ext}_{\mathcal{K}^{*}}(k)=\left\{f(x) \in K^{*}: x \in \operatorname{Ext}_{\mathcal{K}^{*}}(\alpha)\right\}$, and so together with the assumption $\operatorname{Ext}_{\mathcal{K}^{*}}(\alpha)=\operatorname{Ext}_{\mathcal{K}}(\alpha)$, this makes it clear that $\operatorname{Ext}_{\mathcal{K}^{*}}(k)=\operatorname{Ext}_{\mathcal{K}}(k)$. Therefore, if $\mathcal{K}$ is well-founded, and $\lambda$ is strongly inaccessible in $\mathcal{K}, \operatorname{WF}\left(\mathcal{K}^{*}\right)=$ $K(\lambda)=\mathrm{WF}\left(\mathcal{K}^{*}(\lambda)\right)$.

We are now ready to present the proof of Theorem A. Recall that $\mathcal{M}$ is definably well-founded if every element of $\mathcal{M}$ that is first order definable in $\mathcal{M}$ (without 
parameters) is in the well-founded part of $\mathcal{M}$. In particular, if $\mathcal{M}$ is definably wellfounded, then for any parameter-free definable $\alpha \in \operatorname{Ord}^{\mathcal{M}}$, the predecessors of $\alpha$ form a well-ordered set as viewed externally, and thus a nonstandard ordinal of $\mathcal{M}$ (if any) dwarfs any definable ordinal of $\mathcal{M}$. In particular a definably well-founded model is an $\omega$-model. We say that $\mathcal{M}$ is cofinally condensable if the collection of $\alpha \in \operatorname{Ord}^{\mathcal{M}}$ such that $\mathcal{M} \cong \mathcal{M}(\alpha) \prec \mathbb{L}_{\mathcal{M}} \mathcal{M}$ is unbounded in $\operatorname{Ord}^{\mathcal{M}}$.

Theorem A. Assuming that ZFC + "there exists an inaccessible cardinal" has a wellfounded model, there is a model $\mathcal{M}$ of ZFC that is both definably well-founded and cofinally condensable.

Proof The proof is carried out in two steps, the first takes place within an appropriately chosen model $\mathcal{K}$ of ZFC; the second step is performed outside of $\mathcal{K}$.

Step 1. If the theory ZFC + "there exists an inaccessible cardinal" has a well-founded model, then by the Löwenheim-Skolem theorem and the fact that ZF proves that $\mathrm{GCH}$ holds in the constructible universe, there is a countable well-founded model that contains a strongly inaccessible cardinal (since if $\lambda$ is inaccessible in a model $\mathcal{K}$, then $\lambda$ is also inaccessible in the constructible universe $\mathrm{L}^{\mathcal{K}}$ of $\mathcal{K}$; and under GCH every inaccessible cardinal is strongly inaccessible). Let $\mathcal{K}$ be a countable well-founded model that contains a "cardinal" $\lambda$ that is strongly inaccessible in the sense of $\mathcal{K}$. By collapsing $\mathcal{K}$ we may assume that $\mathcal{K}=(K, \in)$. By Remark 2.4 and Lemma 3.2 we can get hold of elements $s$ and $u$ of $K$ satisfying the following conditions:

(i) $\mathcal{K} \models$ "s is a separative $\infty$-satisfaction class for $(\mathrm{V}(\lambda), \in)$ ".

(ii) $\mathcal{K} \models$ " $u$ is unbounded in $\lambda$ and $\forall \delta \in u(\mathrm{~V}(\delta), \in, s \cap \mathrm{V}(\delta)) \prec(\mathrm{V}(\lambda), \in, s)$ ".

Step 2. By Theorem 3.3 and Remark 3.4, there is an elementary extension $\mathcal{K}^{*}$ of $\mathcal{K}$ such that $\operatorname{WF}\left(\mathcal{K}^{*}\right)=K(\lambda)=\operatorname{WF}\left(\mathcal{K}^{*}(\lambda)\right)$.

We claim that $\mathcal{K}^{*}(\lambda)$ is definably well-founded and cofinally condensable. $\mathcal{K}^{*}(\lambda)$ is definably well-founded since $\mathcal{K}(\lambda) \prec \mathcal{K}^{*}(\lambda)$, and $\mathcal{K}(\lambda)$ is well-founded. Recall that $\mathcal{K}$ thinks that $u$ is an unbounded subset of $\lambda$. Since $\mathcal{K} \prec \mathcal{K}^{*}$, to verify that $\mathcal{K}^{*}(\lambda)$ is cofinally condensable it suffices to show that if $\mathcal{K}^{*} \models \delta \in u$, and $\delta \in K^{*} \backslash K$ (equivalently: $\delta$ is a nonstandard element of $\operatorname{Ext}_{\mathcal{K}^{*}}(u)$ ), then $\mathcal{K}^{*}(\lambda) \cong \mathcal{K}^{*}(\delta)$. This is precisely where Lemma 3.1 comes into the picture. If $S:=\operatorname{Ext}_{\mathcal{K}^{*}}(s)$, then $(i)$ and $(\mathrm{ii})$ assure us that the assumptions of Lemma 3.1 are satisfied if we choose any nonstandard ordinal $\tau_{0}$ below $\delta$ and let:

$\mathcal{M}:=\mathcal{K}^{*}(\lambda), \mathcal{N}:=\mathcal{K}^{*}(\delta) ; \tau_{M}:=\tau_{0}, \tau_{N}:=\tau_{0}, S_{M}:=S$ and $S_{N}:=S \cap K^{*}(\delta)$. Hence by Lemma $3.1, \mathcal{K}^{*}(\lambda) \cong \mathcal{K}^{*}(\delta)$, thus concluding the proof of Theorem A.

Remark 3.5 After seeing Theorem A Corey Switzer asked the author whether there are uncountable definably well-founded cofinally condensable models of set theory. Assuming the existence of a weakly compact cardinal, the answer is in the positive. Here we outline the construction of such a model. Let $\lambda$ be a weakly compact cardinal. Then if $T$ is a theory formulated in the infinitary logic $\mathbb{L}_{\lambda, \lambda}$ such that $|T|=\lambda$, and every subset of $T$ of cardinality less than $\lambda$ has a model, then $T$ has a model. This property of $\lambda$ can be used to show that if $X \subseteq \mathrm{V}(\lambda)$, then the model $(\mathrm{V}(\lambda), \in, X)$ has

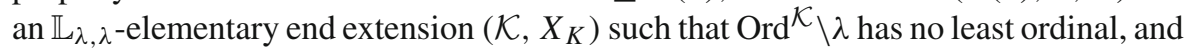


thus $\mathrm{WF}(\mathcal{K})=\mathrm{V}(\lambda)$. In particular, $\mathcal{K}$ is definably well-founded. In order to ensure that $\mathcal{K}$ is also cofinally condensable, we can choose $X$ to be the $\mathbb{L}_{\lambda, \lambda}$ satisfaction class for $(\mathrm{V}(\lambda), \in)$. We then prove a suitable adaptation of Lemma 3.1, where the countability assumption of Lemma 3.1 is replaced with the assumption that both $\mathcal{M}$ and $\mathcal{N}$ have cardinality $\lambda$ for some strongly inaccessible $\lambda, \operatorname{WF}(\mathcal{M})=\mathrm{WF}(\mathcal{N})=\mathrm{V}(\lambda)$, and there are $S_{M} \subseteq M, \tau_{M} \in \operatorname{Ord}^{\mathcal{M}}, S_{N} \subseteq M$, and $\tau_{N} \in \operatorname{Ord}^{\mathcal{N}}$ such that $\tau_{M}$ and $\tau_{N}$ are respectively nonstandard cardinals of $\mathcal{M}$ and $\mathcal{N}, S_{M}$ satisfies Tarski's compositional axioms over $\mathcal{M}$ for all formulae of the $\operatorname{logic} \mathbb{L}_{\delta, \delta}^{\mathcal{M}}$ for $\delta=\tau_{M}$, and $S_{N}$ is satisfies Tarski's compositional axioms over $\mathcal{N}$ for all formula of the logic $\mathbb{L}_{\delta, \delta}^{\mathcal{N}}$ for $\delta=\tau_{N}$. Thus $S_{M}$ correctly calculates the truth value of all $\mathbb{L}_{\lambda, \lambda}$ sentences over $\mathcal{M}$, and $S_{N}$ correctly calculates the truth value of all $\mathbb{L}_{\lambda, \lambda}$ sentences over $\mathcal{N}$. Using an argument very similar to the proof of Theorem $\mathrm{A}$, one then shows that $\mathcal{K}$ is also cofinally condensable.

\section{Proof of Theorem B}

We first lay out a series of definitions and lemmas before presenting the proof of Theorem B.

- Throughout the section, $\mathcal{M}$ is assumed to be a nonstandard model of $\mathrm{ZF}$ and $W:=\operatorname{WF}(\mathcal{M})$.

Definition 4.1 A structure $\mathcal{N}$ is $\operatorname{Cod}_{W}(\mathcal{M})$-saturated if for every type $p\left(x, y_{1}, \cdots, y_{k}\right)$, and for every $k$-tuple $\bar{a}$ of parameters from $\mathcal{N}, p(x, \bar{a})$ is realized in $\mathcal{N}$ provided the following three conditions are satisfied:

(i) $p(x, \bar{y}) \subseteq \mathbb{L}_{\mathcal{M}}$.

(ii) $p(x, \bar{y}) \in \operatorname{Cod}_{W}(\mathcal{M})$.

(iii) $\forall w \in W \mathcal{N} \models \exists x\left(\bigwedge_{\varphi \in p(x, \bar{y}) \cap w} \varphi(x, \bar{a})\right)$.

- In the interest of concision, we say that $\mathcal{M}$ is $W$-saturated if $\mathcal{M}$ is $\operatorname{Cod}_{W}(\mathcal{M})$ -saturated.

Remark 4.1.1. It is not hard to see that if $\mathcal{M}$ is $\omega$-nonstandard, then $\mathcal{M}$ is $W$-saturated iff $\mathcal{M}$ is recursively saturated. We should also point out that a notion closely related to $\operatorname{Cod}_{W}(\mathcal{M})$-saturation was introduced in Ressayre's paper [14] (dubbed $\alpha$-recursive saturation) where it was used as a tool for studying the model theory of admissible languages, as well as certain aspects of descriptive set theory (see also Barwise [1, p.143], Schlipf [16, p.164-165]). A trick similar to the one that shows that recursive saturation coincides with $W$-saturation for $\omega$-nonstandard models can be used to show that, more generally, if $\mathcal{M}$ is nonstandard, then $\mathcal{M}$ is $W$-saturated iff $\mathcal{M}$ is o( $\mathcal{M})$ recursively saturated.

Lemma 4.2 If $\gamma$ is a limit ordinal of $\mathcal{M}$ and $\gamma$ is nonstandard, then $\mathcal{M}(\gamma)$ is $W$ saturated.

Proof Given a 1-type $p(x, \bar{a})$, where $\bar{a}$ is a $k$-tuple of parameters from $\mathcal{M}(\gamma)$ such that conditions $(i),(i i)$, and $(i i i)$ of Definition 4.1 hold, choose $c \in M(\gamma)$ such that 
$p(x, \bar{y})=\operatorname{Ext}_{\mathcal{M}(\gamma)}(c) \cap W$, and let $\theta(z, \bar{y})$ be the following formula in the language of set theory augmented with a predicate $\mathrm{S}$ :

$$
\exists x\left[\forall \varphi(x, \bar{y}) \in c \cap \mathrm{V}(z) \mathrm{S}\left(\varphi\left(c_{x}, c_{y_{1}}, \ldots, c_{y_{k}}\right)\right] .\right.
$$

By Remark 2.4 there is some $s \in M$ such that $S:=\operatorname{Ext}_{\mathcal{M}}(s)$ is a separative $\gamma$-satisfaction class on $\mathcal{M}(\gamma)$. Since for all $\alpha \in \mathrm{o}(\mathcal{M}),(\mathcal{M}(\gamma), S)$ satisfies $\theta(\alpha, \bar{a})$ by Proposition 2.3, $\theta\left(\gamma^{\prime}, \bar{a}\right)$ holds in $(\mathcal{M}(\gamma), S)$ for some $\gamma^{\prime} \in \operatorname{Ord}^{\mathcal{M}(\gamma)} \backslash W$ by Proposition 2.6 (Overspill), which makes it evident that $p(x, \bar{a})$ is realized in $\mathcal{M}(\gamma)$. Note that a slight modification of the proof shows that, more generally, any structure that "lives" in $\mathcal{M}$ (i.e., has an isomorphic copy that is coded in $\mathcal{M}$ ) is $\operatorname{Cod}_{W}(\mathcal{M})$ saturated.

Lemma 4.3 Given countable nonstandard models $\mathcal{M}$ and $\mathcal{N}$ of $\mathrm{ZF}, \mathcal{M} \cong \mathcal{N}$ provided the following two conditions hold:

(a) $\mathcal{M}$ and $\mathcal{N}$ have the same well-founded part $W, \operatorname{Cod}_{W}(\mathcal{M})=\operatorname{Cod}_{W}(\mathcal{M})$, and $\mathrm{ZF}(\mathbb{L}) \subseteq \mathrm{Th}_{\mathbb{L}}(\mathcal{M})=\mathrm{Th}_{\mathbb{L}}(\mathcal{N})$ for $\mathbb{L}:=\mathbb{L}_{\mathcal{M}}=\mathbb{L}_{\mathcal{N}}$.

(b) Both $\mathcal{M}$ and $\mathcal{N}$ are $W$-saturated.

Proof This lemma is a distillation of Lemma 3.1 since the proof of Claim 3.1.1 can be readily modified to show that Claim 3.1.1 holds with the assumptions of Lemma 4.3 once we make the observation that the $W$-saturation of $\mathcal{M}$ implies that the $\mathbb{L}$-type of any finite tuple in $\mathcal{M}$ is a $\operatorname{member} \operatorname{Cod}_{W}(\mathcal{M})$ (and of course the same goes for $\mathcal{N})$. To verify this observation, first consider the following type $p(x, \bar{y})$ :

$$
p(x, \bar{y}):=\left\{\varphi(\bar{y}) \leftrightarrow(\varphi(\bar{y}) \in x): \varphi\left(y_{1}, \ldots, y_{n}\right) \in \mathbb{L}\right\} .
$$

It is easy to see that $p(x, \bar{y}) \in \operatorname{Cod}_{W}(\mathcal{M})$. Given $\bar{a} \in M^{n}$ and $\alpha \in \mathrm{o}(\mathcal{M})$, for sufficiently large $\beta \in \mathrm{o}(\mathcal{M})$, we have:

For all $\varphi(\bar{y}) \in M(\alpha), \mathcal{M} \models \varphi(\bar{a})$ iff $\mathcal{M} \models \operatorname{Sat}_{\beta}(\varphi(\bar{a}))$.

Together with Proposition 2.5 and the assumption that $\mathcal{M} \models \mathrm{ZF}(\mathbb{L})$ we conclude that for each $\alpha \in \mathrm{o}(\mathcal{M})$ the set $\{\varphi(\bar{y}) \in M(\alpha): \mathcal{M} \models \varphi(\bar{a})\}$ is coded in $\mathcal{M}$. This makes it evident that the three conditions of Definition 4.1 are met and therefore by the assumption of $W$-saturation of $\mathcal{M}$, there is an element $c \in M$ such that for all $n$-ary $\mathbb{L}$-formulae $\varphi(\bar{y}) \in \mathbb{L}$, we have:

$$
\mathcal{M} \models \varphi(\bar{a}) \text { iff } \varphi(\bar{y}) \in \operatorname{Ext}_{\mathcal{M}}(c),
$$

which shows that the $\mathbb{L}$-type of $\bar{a}$ is a member of $\operatorname{Cod}_{W}(\mathcal{M})$.

Lemma 4.4 Suppose there is an unbounded collection of $\alpha \in \operatorname{Ord}^{\mathcal{M}}$ such that $\mathcal{M}(\alpha) \prec_{\mathbb{L}_{\mathcal{M}}} \mathcal{M}$. Then $\mathcal{M}$ is $W$-saturated.

Proof This directly follows from Lemma 4.2 and Proposition 2.7 (Elementary Chains).

Lemma 4.5 If there is some $\alpha \in \operatorname{Ord}^{\mathcal{M}}$ with $\mathcal{M}(\alpha) \prec_{\mathbb{L}_{\mathcal{M}}} \mathcal{M}$, then $\mathcal{M} \models \mathrm{ZF}\left(\mathbb{L}_{\mathcal{M}}\right)$. 
Proof Since $\mathcal{M}$ is assumed to be a model of $\mathrm{ZF}$, we just need to verify that $\mathcal{M}$ satisfies $\operatorname{Sep}\left(\mathbb{L}_{\mathcal{M}}\right)$ and $\operatorname{Coll}\left(\mathbb{L}_{\mathcal{M}}\right)$. In light of the assumption that $\mathcal{M}(\alpha)$ is an $\mathbb{L}_{\mathcal{M}}$-elementary submodel of $\mathcal{M}$, it suffices to verify that $\mathcal{M}(\alpha)$ satisfies $\operatorname{Sep}\left(\mathbb{L}_{\mathcal{M}}\right)$ and $\operatorname{Coll}\left(\mathbb{L}_{\mathcal{M}}\right)$. To see that $\mathcal{M}(\alpha) \models \operatorname{Sep}\left(\mathbb{L}_{\mathcal{M}}\right)$, suppose $\psi(x) \in \mathbb{L}_{\mathcal{M}}$ (where $\psi(x)$ is allowed to have parameters from $M(\alpha)$ ), and $m \in M(\alpha)$. Consider

$$
K:=\{k \in M: \mathcal{M}(\alpha) \models k \in m \wedge \psi(k)\} .
$$

By Remark 2.4, there is a separative $\alpha$-satisfaction class $S$ on $\mathcal{M}(\alpha)$. Let $K^{\prime}:=\left\{k \in M:(\mathcal{M}(\alpha), S) \models k \in m \wedge \mathrm{S}\left(\psi\left(c_{k}\right)\right)\right\}$.

By Proposition 2.3, $K=K^{\prime}$. On the other hand, since $S$ is separative, $K^{\prime}$ is coded in $\mathcal{M}(\alpha)$. This concludes the proof that $\operatorname{Sep}\left(\mathbb{L}_{\mathcal{M}}\right)$ holds in $\mathcal{M}(\alpha)$. To verify that $\mathcal{M}(\alpha)$ satisfies $\operatorname{Coll}\left(\mathbb{L}_{\mathcal{M}}\right)$, suppose for some $\varphi(x, y) \in \mathbb{L}_{\mathcal{M}}$ and for some $m$ in $M(\alpha)$ we have:

(1) $\mathcal{M}(\alpha) \models \forall x \in m \exists y \varphi(x, y)$.

We need to verify:

(2) $\mathcal{M}(\alpha) \models \exists z \forall x \in m \exists y \in z \varphi(x, y)$.

Define $f(x)$ in $\mathcal{M}$ to be the unique ordinal $\alpha$ that satisfies $\psi(x, \alpha)$, where $\psi(x, \alpha):=\exists y \in \mathrm{V}(\alpha)(\varphi(x, y) \wedge(\forall \beta \in \alpha \forall y \in \mathrm{V}(\alpha) \neg \varphi(x, y)))$.

Note that by (1) $f$ is well-defined in $\mathcal{M}$ for all $x \in m$. To establish (2) it suffices to show:

(3) $\mathcal{M}(\alpha) \models \exists \beta \in$ Ord $\forall x \in m f(x)<\beta$.

Suppose (3) is false, then:

(4) $\mathcal{M}(\alpha) \models \forall \beta \in$ Ord $\exists x \in m f(x) \geq \beta$.

So by the assumption $\mathcal{M}(\alpha) \prec_{\mathbb{M}} \mathcal{M}$, (4) yields:

(5) $\mathcal{M} \models \forall \beta \in \operatorname{Ord} \exists x \in m f(x) \geq \beta$.

Pick $\beta \in \operatorname{Ord}^{\mathcal{M}} \backslash \operatorname{Ord}^{\mathcal{M}(\alpha)}$, then by (5) there is some $m_{0} \in \operatorname{Ext}_{\mathcal{M}}(m)\left(=\operatorname{Ext}_{\mathcal{M}}(\alpha)(m)\right)$ such that $f\left(m_{0}\right) \geq \beta$ holds in $\mathcal{M}$. This contradicts $\mathcal{M}(\alpha) \prec \mathbb{L}_{\mathcal{M}} \mathcal{M}$ since $f$ is an $\mathbb{L}_{\mathcal{M}}$-definable function in $\mathcal{M}$, thereby showing the veracity of (3). This concludes the verification of $\operatorname{Coll}\left(\mathbb{L}_{\mathcal{M}}\right)$ in $\mathcal{M}$.

Lemma 4.6 If $\mathcal{M}$ is a nonstandard model of $\mathrm{ZF}\left(\mathbb{L}_{\mathcal{M}}\right)$, and $\mathcal{M}$ is $W$-saturated, then $\mathcal{M}$ is cofinally condensable.

Proof Fix any nonstandard $\gamma \in \operatorname{Ord}^{\mathcal{M}}$ and consider the type $p(x, \gamma)$ (where $\gamma$ is treated as a parameter) consisting of the formula $(\gamma \in x) \wedge \operatorname{Ord}(x)$ together with formulae of the form $\operatorname{Ref}_{\varphi}(x)$ (as in Proposition 2.8) as $\varphi$ ranges in $\mathbb{L}_{\mathcal{M}}$. It is easy to see that $p(x, y)$ satisfies conditions $(i)$ and $(i i)$ of Definition 4.1. Moreover, by Proposition 2.8 (Reflection) $p(x, \gamma)$ also satisfies condition (iii) of Definition 4.1. Therefore by the assumption of $W$-saturation of $\mathcal{M}, p(x, \gamma)$ is realized in $\mathcal{M}$ by some $\gamma^{\prime}$, which makes it clear that $\gamma^{\prime}$ is above $\gamma, \gamma^{\prime}$ is a nonstandard limit ordinal of $\mathcal{M}$, and $\mathcal{M}\left(\gamma^{\prime}\right) \prec_{\mathbb{L}_{\mathcal{M}}} \mathcal{M}$. Thanks to Lemmas 4.2 and $4.3, \mathcal{M}\left(\gamma^{\prime}\right) \cong \mathcal{M}$, thus $\mathcal{M}$ is cofinally condensable.

We are now ready to establish Theorem $B$. The special case of Theorem B for countable $\omega$-nonstandard models of ZF follows from Schlipf's work on recursively saturated models of ZF in [17] and [18].

Theorem B. The following are equivalent for a countable model $\mathcal{M}$ of $\mathrm{ZF}$. 
(a) $\mathcal{M}$ is condensable.

(b) $\mathcal{M}$ is cofinally condensable.

(c) $\mathcal{M}$ is nonstandard, and $\mathcal{M}(\alpha) \prec_{\mathbb{M}} \mathcal{M}$ for an unbounded collection of $\alpha \in$ Ord $^{\mathcal{M}}$.

(d) $\mathcal{M}$ is nonstandard and $W$ - saturated, and $\mathcal{M} \models \mathrm{ZF}\left(\mathbb{L}_{\mathcal{M}}\right)$.

(e) For some nonstandard ordinal $\gamma$ of $\mathcal{M}$ and some $S \subseteq M, S$ is an amenable $e^{4} \gamma$ satisfaction class on $\mathcal{M}$.

Proof We will first show the equivalence of $(a),(b)$, and $(c)$ by establishing $(b) \Rightarrow$ $(a) \Rightarrow(c) \Rightarrow(b)$. This will allow us to show the equivalence of $(d)$ with each of $(a)$, $(b)$, and $(c)$ by proving $(a) \Rightarrow(d) \Rightarrow(a)$. Finally, we demonstrate $(a) \Rightarrow(e) \Rightarrow(d)$ to complete the proof.

(b) $\Rightarrow$ (a). Trivial.

(a) $\Rightarrow$ (c). Suppose $\mathcal{M}$ is condensable with $\mathcal{M} \cong \mathcal{M}(\alpha) \prec_{\mathbb{L}_{\mathcal{M}}} \mathcal{M}$. Then by "unwinding" the isomorphism between $\mathcal{M}$ and $\mathcal{M}(\alpha)$, we can readily obtain a sequence of models $\left\langle\mathcal{N}_{n}: n \in \omega\right\rangle$ such that $\mathcal{N}_{0}=\mathcal{M}$ and for all $n \in \omega$ the following hold:

(1) $\mathcal{N}_{n}=\left(\mathrm{V}\left(\alpha_{n}\right), \in\right)^{\mathcal{N}_{n+1}}$ for some $\alpha_{n} \in \operatorname{Ord}\left(\mathcal{N}_{n+1}\right)$.

(2) $\mathcal{N}_{n} \prec \mathbb{L} \mathcal{N}_{n+1}$, where $\mathbb{L}:=\mathbb{L}_{\mathcal{M}}$.

(3) $\mathcal{N}_{n} \cong \mathcal{M}$.

Let $\mathcal{N}:=\bigcup_{n \in \omega} \mathcal{N}_{n}$. By Proposition 2.7 (Elementary Chains), $\mathcal{N}_{n} \prec \mathbb{L} \mathcal{N}$ for all $n \in \omega$, which together with Lemma 4.4 implies that $\mathcal{N}$ is $W$-saturated, where $W=$ $\mathrm{WF}(\mathcal{N})=\mathrm{WF}\left(\mathcal{N}_{n}\right)$ for all $n \in \omega$. By Lemma $4.2, \mathcal{M}$ is also $W$-saturated. Therefore $\mathcal{N} \cong \mathcal{M}$ by Lemma 4.3, which in light of (2) and (3) and the unboundedness of $\left\{\alpha_{n}: n \in \omega\right\}$ in $\operatorname{Ord}^{\mathcal{N}}$ makes it clear that (c) holds.

(c) $\Rightarrow$ (b). Assume (c). It is easy to see, using Proposition 2.6 and Lemma 4.2, that $\mathcal{M}$ is $W$-saturated. By (c) we can choose a nonstandard $\gamma \in \operatorname{Ord}^{\mathcal{M}}$ arbitrarily high in $\operatorname{Ord}^{\mathcal{M}}$ such that $\mathcal{M}_{\gamma} \prec_{\mathbb{L}_{\mathcal{M}}} \mathcal{M}$. Since by Lemma $4.2 \mathcal{M}_{\gamma}$ is $W$-saturated, we can now invoke Lemma 4.3 to conclude that $\mathcal{M} \cong \mathcal{M}_{\gamma} \prec \mathbb{L}_{\mathcal{M}} \mathcal{M}$, which makes it evident that (b) holds.

(a) $\Rightarrow$ (d). If $\mathcal{M} \cong \mathcal{M}(\alpha) \prec \mathbb{L}_{\mathcal{M}} \mathcal{M}$ for some $\alpha \in \operatorname{Ord}(\mathcal{M})$, then $\mathcal{M}$ is clearly nonstandard. Moreover, $\mathcal{M}$ is $W$-saturated by Lemma 4.2; and $\mathcal{M}$ satisfies $\mathrm{ZF}\left(\mathbb{L}_{\mathcal{M}}\right)$ by Lemma 4.5.

(d) $\Rightarrow$ (a). This is justified by Lemma 4.6.

(a) $\Rightarrow$ (e). Suppose (a) holds and let $\alpha \in \operatorname{Ord}(\mathcal{M})$ such that $\mathcal{M} \cong \mathcal{M}(\alpha) \prec_{\mathbb{L}_{\mathcal{M}}} \mathcal{M}$. By Proposition 2.5 for each $\delta \in \mathrm{o}(\mathcal{M})$, there is some $S \in M$ such that $S$ is a $\delta$ satisfaction predicate over $\mathcal{M}(\alpha)$ that is definable in $\mathcal{M}(\alpha)$ by an $\mathbb{L}_{\mathcal{M}(\alpha)}$-formula. Since we have verified that $(a) \Rightarrow(d), \mathcal{M}(\alpha)$ satisfies $\mathrm{ZF}\left(\mathbb{L}_{\mathcal{M}}\right)$, which assures us that $S$ is an amenable $\delta$-satisfaction predicate over $\mathcal{M}(\alpha)$. Proposition 2.6 (Overspill) can be readily invoked (applied to $\mathcal{M}$, rather than $(\mathcal{M}, S)$ ) to show there is some nonstandard $\gamma$ in $\mathcal{M}$ such that $\mathcal{M}$ satisfies "there is an amenable $\gamma$-satisfaction class over $(\mathrm{V}(\alpha), \in)$ " . In light of the assumption that $\mathcal{M} \cong \mathcal{M}(\alpha)$, this shows that (e) holds.

To carry out the overspill argument, we will distinguish between the case when $\mathcal{M}$ is $\omega$-standard, and the case when $\mathcal{M}$ is $\omega$-nonstandard. If $\mathcal{M}$ is $\omega$-standard, the

4 This notion was defined in Definition 2.2. 
overspill argument succeeds smoothly since by routine absoluteness considerations, for $s \in M$ and $S:=\operatorname{Ext}_{\mathcal{M}}(s)$, we have:

$S$ is amenable over $\mathcal{M}(\alpha)$ iff $\mathcal{M} \models$ "s is amenable over $(\mathrm{V}(\alpha), \in)$ ".

However, since the left-to-right direction of the above equivalence can break down for $\omega$-nonstandard models (e.g., for models of $\mathcal{M}$ of ZF that satisfy $\neg \operatorname{Con}(\mathrm{ZF})$ ) we will spell out the overspill argument for the case that $\mathcal{M}$ is $\omega$-nonstandard in more detail. It is worth pointing out that in this case (a) implies that $\mathcal{M}$ is recursively saturated, and by the resplendence property of countable recursively saturated models one can readily conclude that $\mathcal{M}$ carries an amenable $j$-satisfaction class for some nonstandard $j \in \omega^{\mathcal{M}}$. However, the overspill argument we present establishes (e) without the assumption of countability of $\mathcal{M}$. Within $\mathcal{M}$, for each $i \in \omega^{\mathcal{M}}$ let $\operatorname{Repl}_{i}(\mathrm{~S})$ consist of all instances of the replacement scheme in the language $\{\in, S\}$ whose length is at most $i$. Then define a subset $S$ of $\mathrm{V}(\alpha)$ to be $i$-amenable over $(\mathrm{V}(\alpha), \in)$ if $(\mathrm{V}(\alpha), \in, S) \models \operatorname{Repl}_{i}(\mathrm{~S})$. Since $\mathcal{M}(\alpha)$ is a model of ZF and for each "real world" natural number $n$, there is an $n$-satisfaction class over $\mathcal{M}(\alpha)$ that is first order definable in $\mathcal{M}$, we may conclude:

(1) For each $n \in \omega \mathcal{M} \models$ " $\exists s$ ( $s$ is $n$-amenable over $(\mathrm{V}(\alpha), \epsilon)$ )" .

Therefore by Overspill, there is some nonstandard $j \in \omega^{\mathcal{M}}$ such that:

(2) $\mathcal{M} \models$ " $\exists s$ ( $s$ is $j$-amenable over $(\mathrm{V}(\alpha), \in))$ ".

Let $s \in M$ be a witness to the existential statement in (2) and $S:=\operatorname{Ext}_{\mathcal{M}}(s)$. It is evident that $S$ is an amenable $j$-satisfaction class over $\mathcal{M}(\alpha)$, as desired.

(e) $\Rightarrow(\mathbf{d})$. The $W$-saturation of $\mathcal{M}$ is readily verifiable with a reasoning very similar to the proof of Lemma 4.2. To see that $\mathcal{M} \models \mathrm{ZF}\left(\mathbb{L}_{\mathcal{M}}\right)$, it is sufficient to verify that the replacement scheme holds in $\mathcal{M}$ for all $\mathbb{L}_{\mathcal{M}}$-formulae. To this end, let $\varphi(x, y)$ be an $\mathbb{L}_{\mathcal{M}}$-formula (possibly with parameters from $\mathcal{M}$ ), and suppose that $\mathcal{M} \models$ $\forall x \exists ! y \varphi(x, y)$. Let $f: M \rightarrow M$ be the function whose graph is described by $\varphi$. Given $c \in M$, we want to show that there is $d \in M$ such that

$$
\operatorname{Ext}_{\mathcal{M}}(d)=\left\{f(m): m \in \operatorname{Ext}_{\mathcal{M}}(c)\right\}
$$

By Proposition 2.3 the graph of $f$ is also given by the formula $\theta(x, y, \varphi):=$ $\mathrm{S}\left(\varphi\left(c_{x}, c_{y}\right)\right)$ (where $\varphi$ is treated as a parameter). The assumption that $(\mathcal{M}, S) \models$ $\operatorname{Repl}(\mathrm{S})$ then allows us to get hold of $d \in M$ such that $\operatorname{Ext}_{\mathcal{M}}(d)=\{f(m): m \in$ $\left.\operatorname{Ext}_{\mathcal{M}}(c)\right\}$.

Remark 4.7 An examination of the proof of Theorem B makes it clear that the following implications hold without the assumption of countability of $\mathcal{M}$ :

$$
(b) \Rightarrow(a) \Rightarrow(e) \Rightarrow(c) \Leftrightarrow(d) \text {. }
$$

We suspect that the implication $(a) \Rightarrow(b)$ fails for some uncountable model of ZF, but we have not been able to verify this. However, the remaining two implications can be shown to be irreversible by resorting to well-known uncountable models, as we shall explain.

The failure of $(e) \Rightarrow(a)$ is illustrated by the easily verified fact that there are $\omega_{1}$-like recursively saturated models of $\mathrm{ZF}$ : start with a countable recursively saturated model 
$\mathcal{M}_{0}$ of ZF and let $S_{0}$ be an amenable $j$-satisfaction class $S_{0}$ for some nonstandard $j \in \omega^{\mathcal{M}}$. Then use the Keisler-Morley Theorem ${ }^{5}$ to build an $\omega_{1}$-like elementary end extension $(\mathcal{M}, S)$ of $\left(\mathcal{M}_{0}, S_{0}\right)$. It is evident that $\mathcal{M}$ is recursively saturated but not condensable.

The failure of $(c) \Rightarrow(e)$ is illustrated by the fact that there are $\omega_{1}$-like rather classless recursively saturated models of ZF; this fact was first established by Matt Kaufmann [13] using the combinatorial principle $\diamond_{\omega_{1}}$; later Shelah [19] used an absoluteness argument to eliminate $\nabla_{\omega_{1}}$ (but no "direct proof" of this fact has been yet discovered). Note that by Tarski's theorem on undefinability of truth, a rather classless model cannot even carry a separative $\gamma$-satisfaction class for nonstandard $\gamma{ }^{6}$

It is also worth pointing out that the equivalence of condition (a) through (d) of Theorem B holds for models of ZF of arbitrary cardinality provided "condensable" is replaced by "weakly condensable", where $\mathcal{M}$ is said to be weakly condensable if $\mathcal{M} \cong{ }_{p} \mathcal{M}(\alpha) \prec_{\mathbb{L}_{\mathcal{M}}} \mathcal{M}$ for some $\alpha \in \operatorname{Ord}(\mathcal{M})$; here $\cong_{p}$ denotes partial isomorphism (two relational structures are said to be partially isomorphic if there is a nonempty family of partial isomorphisms between them that has the back-and-forth property, see [1]).

Remark 4.8 Condensability is a robust notion, as indicated by (1) and (2) below.

(1) It is easy to see, using the definition of condensability, that condensability is inherited by inner models (by an inner model of a model $\mathcal{M}$ of ZF here we mean a transitive subclass of $\mathcal{M}$ that satisfies $\mathrm{ZF}$, contains all the ordinals of $\mathcal{M}$, and is definable in $\mathcal{M}$ by an $\mathbb{L}_{\mathcal{M}}$-formula).

(2) The equivalence of (a) and (c) of Theorem $B$ can be used to show that if $\mathcal{M}$ is a condensable model of $\mathrm{ZF}$, and $\mathbb{P}$ is set-notion offorcing in $\mathcal{M}$, then for every $\mathbb{P}$-generic filter $G$ over $\mathcal{M}, \mathcal{M}[G]$ is also condensable (the proof is similar to the special case when $\mathcal{M}$ is recursively saturated, as in the proof of Theorem 2.6 of [5]). The situation is quite different for class notions of forcing, since as shown in Theorem 2.8 of [6] every countable model of ZF has a class-generic extension to a Paris model of ZF, and of course no Paris model is condensable.

Acknowledgements I am grateful to Paul Kindvall Gorbow, Zachiri McKenzie, and Jim Schmerl for their feedback on earlier versions of this paper. Special thanks also go to the referee for a most helpful report.

Funding Open access funding provided by University of Gothenburg. The research presented in this article was partially supported by the National Science Centre, Poland (NCN), grant number 2019/34/A/HS1/00399.

Open Access This article is licensed under a Creative Commons Attribution 4.0 International License, which permits use, sharing, adaptation, distribution and reproduction in any medium or format, as long as you give appropriate credit to the original author(s) and the source, provide a link to the Creative Commons licence, and indicate if changes were made. The images or other third party material in this article are included in the article's Creative Commons licence, unless indicated otherwise in a credit line to the material. If

\footnotetext{
5 The Keisler-Morley Theorem is often stated for countable models of ZF, but the usual omitting types proof of the theorem (as in [3, Theorem 2.2.18]) works for equally well for all countable models $(\mathcal{M}, S)$, where $\mathcal{M}$ is a model of $\mathrm{ZF}$ and $S$ is amenable over $\mathcal{M}$.

6 Indeed by a theorem of Smith [20], no rather classless model $\mathcal{M}$ carries a $j$-satisfaction class for any nonstandard $j \in \omega^{\mathcal{M}}$. Smith's result was formulated for models of PA, but his proof works equally well for models of ZF.
} 
material is not included in the article's Creative Commons licence and your intended use is not permitted by statutory regulation or exceeds the permitted use, you will need to obtain permission directly from the copyright holder. To view a copy of this licence, visit http://creativecommons.org/licenses/by/4.0/.

\section{References}

1. Barwise, J.: Admissible sets and structures, perspectives in mathematical logic. Springer, Verlag (1975)

2. Barwise, J., Schlipf, J.: An introduction to recursively saturated and resplendent models. J. Sym. Logic 41, 531-536 (1976)

3. Chang, C.C., Keisler, H.J.: Model Theory, 3rd edn. North-Holland publishing Co., Amsterdam (1990)

4. Enayat, A.: On certain elementary extensions of models of set theory. Trans. Amer. Math. Soc. 283, 705-715 (1984)

5. Enayat, A.: Counting models of set theory. Fund. Math. 174, 23-47 (2002)

6. Enayat, A.: Models of set Theory with definable ordinals. Arch. Math. Logic 44, 363-385 (2005)

7. Enayat, A., McKenzie, Z.: Initial embeddings of models of set theory, under review (2019). Available online: http://front.math.ucdavis.edu/1906.02873

8. Friedman, H.: Countable models of set theories. Lecture Notes in Mathematics, vol. 337, pp. 539-573. Springer, Berlin (1973)

9. Gorbow, P.K.: Self-similarity in the Foundations, Doctoral Dissertation, University of Gothenburg (2018). Available online: arXiv: 1806.11310

10. Gorbow, P.K.: Rank-initial embeddings of non-standard models of set theory. Arch. Math. Logic 59, 517-563 (2020)

11. Hutchinson, J.: Elementary extensions of countable models of set theory. J. Sym. Logic 41, 139-145 (1976)

12. Jech, T.: Set Theory. Springer Monographs in Mathematics. Springer, Berlin (2003)

13. Kaufmann, M.: A rather classless model. Proc. Amer. Math. Soc. 62, 330-333 (1977)

14. Ressayre, J.-P.: Models with compactness properties relative to an admissible language. Ann. Pure Appl. Logic 11, 31-55 (1977)

15. Ressayre, J.-P.: Modèles non standard et sous-systèmes remarquables de ZF, Modéles Non standard en Arithmétique et Théorie des Ensembles, Publications Math ématiques de l'Université Paris VII. Vol. 22. Université de Paris VII, U.E.R. de Mathématiques, Paris, pp. 47-147 (1987)

16. Schlipf, J.: A guide to the identification of admissible sets above structures. Ann. Pure Appl. Logic 12, 151-192 (1977)

17. Schlipf, J.: Toward model theory through recursive saturation. J. Sym. Logic 43, 183-206 (1978)

18. Schlipf, J.: Recursively saturared models of set theory. Proc. Amer. Math. Soc. 80, 135-142 (1980)

19. Shelah, S.: Models with second order properties II: trees with no undefined branches. Ann. Pure Appl. Logic 14, 73-87 (1978)

20. Smith, S.T.: Nonstandard definability. Ann. Pure Appl. Logic 42, 21-43 (1989)

Publisher's Note Springer Nature remains neutral with regard to jurisdictional claims in published maps and institutional affiliations. 